\title{
Underwater endoclip closure after endoscopic resection for duodenal adenomas
}

\author{
Yasushi Yamasaki, Hiromitsu Kanzaki, Yoshiro Kawahara, Hiroyuki Okada
}

Okayama University Hospital, Okayama, Japan

Duodenal endoscopic resection remains challenging because of the high risk of adverse events [1]. Complete endoclip closure of mucosal defects can reduce delayed adverse events [2], but endoclip closure is sometimes difficult because the duodenal lumen is narrow and has a precipitous flexure. Additionally, insufflation during the procedure elevates the intraduodenal pressure and distends mucosal defects, resulting in great difficulty in the achievement of complete endoclip closure. Underwater conditions reportedly reduce the size of mucosal defects after colorectal endoscopic mucosal resection [3]. In practice, we have also found that mucosal defects are small after duodenal endoscopic resection in underwater conditions. Thus, we applied underwater endoclip closure to mucosal defects after duodenal endoscopic resection.

After 20-mm adenomas in the second portion of the duodenum were removed by underwater endoscopic mucosal resection using a $15-\mathrm{mm}$ polypectomy snare (Captivator II; Boston Scientific, Natick, MA) (Fig. 1A), white light and narrow-band imaging endoscopy showed no residue. The mucosal defects were closed completely by endoclips (HX-610-090; Olympus, Tokyo) in four patients (100\%). In underwater conditions, the pressure in the duodenal lumen remains low, which decreases the size of the mucosal defects after duodenal endoscopic resection (Fig. 1B). Additionally, the resection border floated up into the duodenal lumen, allowing us to easily close the mucosal defects after duodenal underwater endoscopic mucosal resection by endoclips in only a few minutes (Fig.1 C,D; Video 1, supplementary material, online version). During all procedures, physiological saline was used

Department of Gastroenterology, Okayama University Hospital,

Okayama, Japan

Conflict of Interest: None

Correspondence to: Yasushi Yamasaki, Department of

Gastroenterology, Okayama University Hospital, 2-5-1 Shikata-cho,

Kita-ku, Okayama-city, Okayama 700-8558, Japan,

e-mail: yasshifive@yahoo.co.jp

Received 11 September 2017; accepted 20 September 2017; published online 16 October 2017

DOI: https://doi.org/10.20524/aog.2017.0202

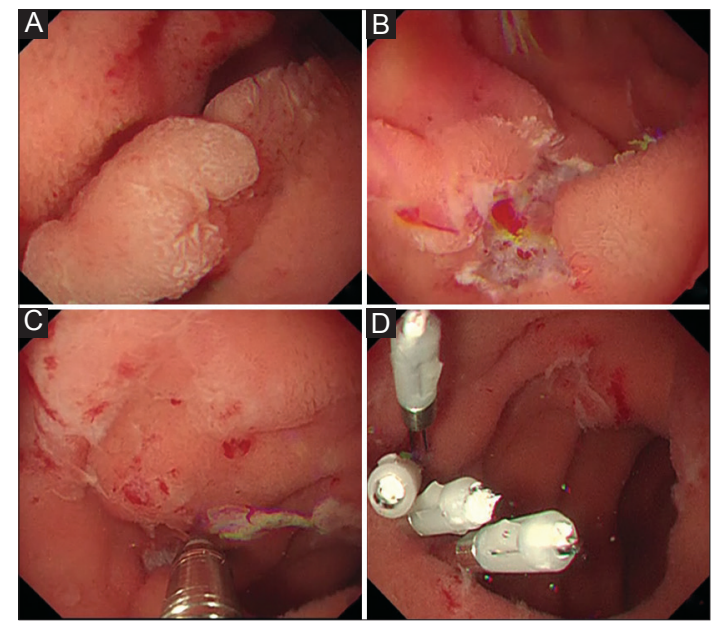

Figure 1 Underwater endoclip closure after duodenal underwater endoscopic mucosal resection. (A) A 20-mm adenoma was located at the second portion of the duodenum. (B) Mucosal defect after piecemeal underwater endoscopic mucosal resection. The resection borders floated upward. (C) Endoclip closure in underwater conditions. (D) Complete closure was achieved

to fill the duodenal lumen, and the air insufflation switch was turned off. No adverse events occurred after the procedure.

\section{Acknowledgment}

We are sincerely grateful to the medical staff in our endoscopy unit.

\section{References}

1. Inoue $\mathrm{T}$, Uedo $\mathrm{N}$, Yamashina $\mathrm{T}$, et al. Delayed perforation: a hazardous complication of endoscopic resection for non-ampullary duodenal neoplasm. Dig Endosc 2014;26:220-227.

2. Yamasaki Y, Takeuchi Y, Uedo N, et al. Line-assisted complete closure of duodenal mucosal defects after underwater endoscopic mucosal resection. Endoscopy 2016;49(S 01):E37-E38.

3. Curcio G, Granata A, Ligresti D, Traina M. Downsizing the target: the underwater closure technique. Endoscopy 2015;47 (Suppl 1): UCTN:E369-E370. 